\title{
Frictional hyperspheres in hyperspace
}

\author{
François Guillard $\odot^{*}$ and Benjy Marks $\odot$ \\ School of Civil Engineering, The University of Sydney, New South Wales 2006, Australia
}

(Received 24 September 2020; revised 20 April 2021; accepted 21 April 2021; published 17 May 2021)

\begin{abstract}
We extend the formulation of the discrete element method, which is typically used to simulate granular media, to describe arbitrarily large numbers of spatial dimensions and the collisions of frictional hyperspheres in these simulations. These higher dimensional simulations require complex visualization techniques, which are also developed here. Under uniaxial compression, we find that the stiffness of a granular medium is independent of the dimension for dimensions greater than one. In the dense flow regime, we show that the compressibility and frictional properties of higher dimensional granular materials can be described by a common rheology, with the main distinction between dimensions being the packing fraction. Results from these simulations extend our understanding of the effects of dimensionality on the behavior of granular materials, and on elastic and frictional properties in higher dimensions.
\end{abstract}

DOI: 10.1103/PhysRevE.103.052901

\section{INTRODUCTION}

Physicists strive to describe the world based on observations made in three spatial dimensions. However, the mathematical tools and the physical laws developed can usually be generalized to higher dimensional spaces. Such generalizations can shed light on the deeper structure of theories and models, and can suggest new approaches to solve $3 \mathrm{D}$ problems, improving our understanding of the world we live in. Several theories therefore rely on higher dimensional spaces such as the Riemannian manifolds in general relativity [1], the potential for our universe to be a hologram [2], multidimensional spaces in string theories [3], and the projection of higher dimensional crystals to explain the structure of quasicrystals in chemistry [4].

As higher dimensional analytical models are developed, and since experimental validation cannot typically be performed, numerical simulations offer a pathway to study higher dimensional behavior. Here we will focus on discrete element modeling, which simulates the motion of assemblies of individual particles based on the forces that govern their interaction. Using similar methods, the higher dimensional packing of hard frictionless spheres has previously received significant attention [5-7] and has been shown to have important consequences in information theory [8] and error recovery for data transmission [9]. Molecules and phase transitions are often simulated with molecular dynamics, which has been extended to a fourth spatial dimension to improve the computational efficiency of protein folding by removing the possibility of knots [10] and accelerate structural calculations in other systems $[11,12]$.

\footnotetext{
*francois.guillard@sydney.edu.au
}

Published by the American Physical Society under the terms of the Creative Commons Attribution 4.0 International license. Further distribution of this work must maintain attribution to the author(s) and the published article's title, journal citation, and DOI.
Previous simulations have dealt only with translational degrees of freedom: the particles do not have any degrees of freedom in rotation [6,13-15]. Granular materials, such as sand, cereal, or snow, are primarily controlled by the friction between their constituent particles. This friction acts to frustrate the rotation of the particles, which in turn dictates their dynamic behavior. Any attempt to study higher dimensional granular materials numerically must therefore include rotational degrees of freedom, which are captured in the simulation scheme presented in this work alongside specific visualization tools.

\section{SIMULATION METHOD}

A hypersphere is the generalization of a sphere to an arbitrary number of dimensions, $N$. A hypersphere is therefore defined as the set of points that are a distance $r$ from its center, and we limit ourselves to Euclidean spaces. The coordinates of the center of a hypersphere are denoted $x_{i}$ along the directions $e_{i}$, with $i$ from 1 to $N$. We use the term hypersphere to denote the surface (or more generally the boundary) of an $N$-ball.

We simulate the motion of frictional hyperspheres using an extension of the discrete element method [16], which computes the translational and rotational motion of individual grains by solving the equations of momentum and angular momentum conservation for each particle. When grains contact one another we model the collision using linear elastic repulsion with elastic constant $k_{n}$ and viscous damping in the normal direction, as well as linear springs, viscous damping, and Coulomb friction in the direction tangential to the contact, with friction coefficient $\mu_{g}$. The inclusion of tangential forces, and in particular friction, is essential in this study as those forces affect the rotational motion of the grains, which is a key aspect in understanding the mechanical behavior of granular media. The simulations are nondimensional, with the diameter of the hyperspheres giving the length unit $[\mathrm{L}]$ and their mass giving the mass unit $[\mathrm{M}]$. The time unit $[\mathrm{T}]$ is set by the exter- 

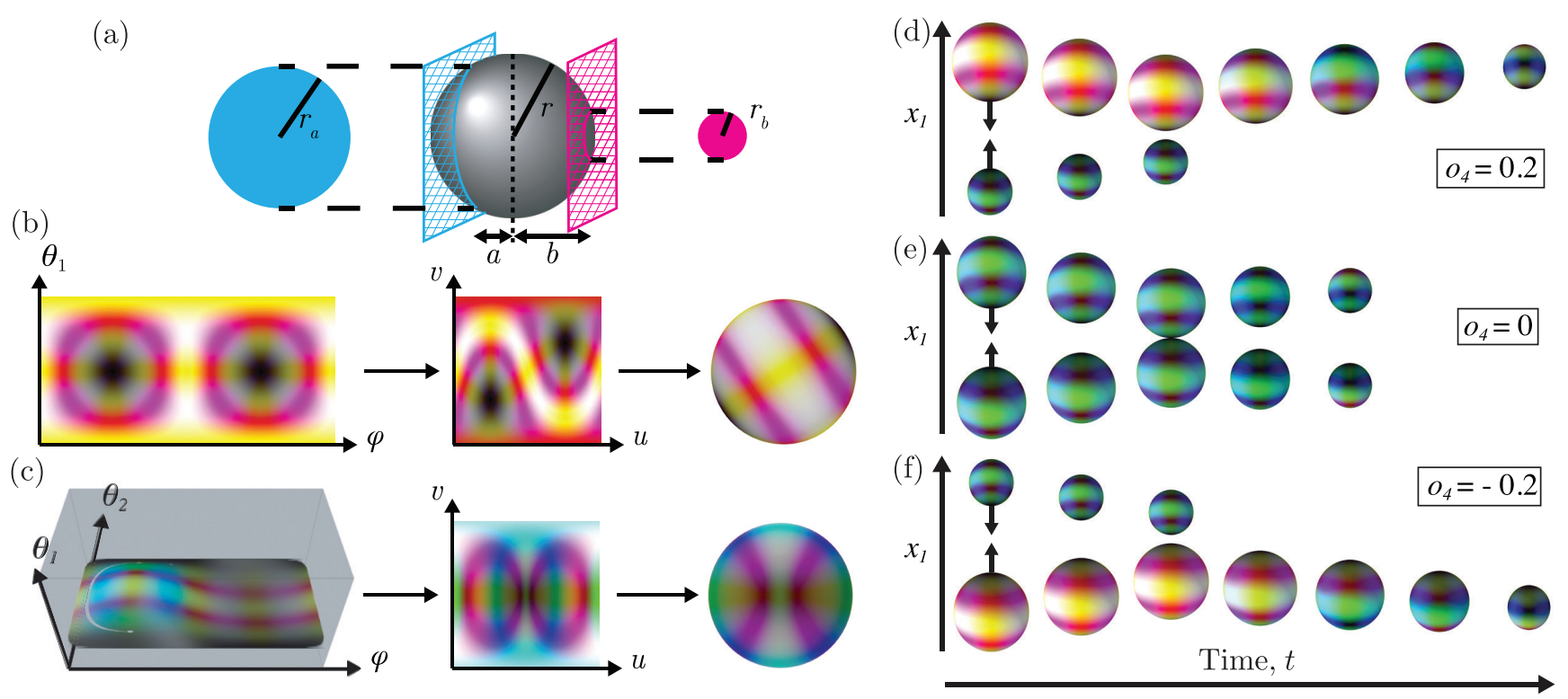

FIG. 1. Visualizing hyperspheres. (a) Schematic representation of a 3-ball (gray) being sliced by two 2D planes (blue and pink) at distances $a$ and $b$ from its center (dashed line). The size of the respective intersecting 2-balls (circles) are shown in blue and pink, with reduced radii $r_{a}$ and $r_{b}$. (b) Texturing process for a 3-ball. Left: 2D source texture in hyperspherical coordinates $\varphi$ and $\theta_{1}$. Center: A set of points on the projected 2D surface of the 3-ball representation of the original 3-ball in $u, v$ coordinates. Parameterization of the rendered sphere surface using $u, v$ coordinates, with corresponding color from the 2D texture. Right: Rendering of the 3-ball with attached texture. (c) Texturing process for a 4-ball. Left: 3D source texture in hyperspherical coordinates $\varphi, \theta_{1}$, and $\theta_{2}$. The gray volume indicates the region in which the $3 \mathrm{D}$ texture volume is defined, and the locations of the displayed colors within it correspond to the points to be mapped from this volume. Center: A set of points on the projected 2D surface of the 3-ball representation of the original 4-ball in $u, v$ coordinates. Parameterization of the rendered sphere surface using $u, v$ coordinates, with corresponding color from the 3D texture. Right: Rendering of the 4-ball with attached texture. [(d)-(f)] Two 4-balls colliding. Particles moving towards each other, except for an offset in $x_{4}$, with particle centers at $x_{4}= \pm 0.2$. (d) Observer with $o_{4}=0.2$, the same as the initial position of the center of the top particle. (e) Observer with $o_{4}=0$, the same as the the contact point. (f) Observer with $o_{4}=-0.2$, the same as the initial position of the center of the bottom particle.

nal gravitational force applied to the grains $\vec{g}=1\left[\mathrm{~L} \mathrm{~T}^{-2}\right]$. See Appendix A for details on the equations of motion and their numerical implementation.

Visualizing simulation results is crucial to understanding the behavior and characteristics of any simulated system. A large variety of techniques can be used to visually examine the dynamics of 2D and 3D granular media [17-20]. For higher dimensional granular media it is not possible to represent all of the particles in a 3D space simultaneously, and the particles must be intersected with a 1D, 2D, or 3D space. A straightforward method to do this is to slice the hyperspace with a 3D volume: all but three of the dimensions are frozen, resulting in a 3D space which can be explored using conventional viewing techniques. By progressively scanning through the frozen values, the higher dimensions can be explored as well. Hyperspheres are thus represented visually as 3-balls, but usually with a smaller diameter than the actual hypersphere. This method of visualization is shown schematically in Fig. 1(a) where a 3-ball is visualized in two dimensions by slicing with a 2D plane. Interactive examples of this visualization technique can be found at Ref. [21] and in a video in the Supplemental Material [22].

With this method, for systems where $N>3$, the first three of the $N$ dimensions are selected as those to intersect with. In order to actually perform the rendering, an observer location $\vec{o}$ needs to be defined. This observer has the property that it renders only objects that have the same coordinates $o_{i}$ for $i>3$ (effectively implementing the intersection with a 3D space discussed previously). The remaining coordinates $o_{1}$, $o_{2}, o_{3}$, are then used to create the final on-screen rendering (2D).

By varying the observer location in higher dimensions, the rendered particles remain at the same apparent location but change in apparent size [cf. Fig. 1(a)]. As an example, the collision of two 4-balls, with a texture to their boundary as a means of visualizing their rotation, is shown in Figs. 1(d)1(f). Two particles move towards each other in $x_{1}$ and are initially aligned along this axis except for an offset of $x_{4}=$ \pm 0.2 . Their contact point is at $x_{4}=0$ [Fig. 1(e)], and the hyperspheres do not appear to contact from other viewing locations [Fig. 1(d) or 1(f)]. After collision, the particles gain velocity in $x_{4}$, and therefore their size observed from a particular $o_{4}$ evolves over time until they eventually disappear from view. At the same time, the particles acquired a rotational velocity from their collision, and therefore their texture also evolves.

In order to visualize the rotation of hyperspheres, we apply suitable textures to them, so that changes in these textures are visible when the particle rotates. The boundary of a $N$-dimensional ball is a $N$-1-dimensional sphere, and so, for dimensions higher than three, only a subset of the full ball boundary texture is mapped onto its 
representation in $3 \mathrm{D}$ space. For the purposes of texturing particles, it is convenient to parameterize this surface using hyperspherical coordinates, such that a point on the surface of the hypersphere can be located using $N-1$ angles $\varphi, \theta_{1}, \theta_{2}, \ldots, \theta_{N-2}$, with the angles $\varphi \in[0,2 \pi]$ and $\theta_{i} \in$ $[0, \pi]$. Using this notation, one can define a "texturing" function which maps these hyperspherical coordinates to a given color space. Moreover, to be a smooth texture the function $f:\left(\varphi, \theta_{1} \ldots \theta_{N-2}\right) \longrightarrow$ (color) should also verify, $\forall \theta, f(\varphi=$ $0)=f(\varphi=2 \pi)$, so that it is periodic when mapped on to the particle, and $\forall k \in\{1: N-2\}, \forall \theta_{i \neq k}, \forall \varphi, f\left(\theta_{k}=0\right)=$ constant and $f\left(\theta_{k}=\pi\right)=$ constant, which ensures that points for which certain $\theta_{i}$ are 0 or $\pi$ are defined by a single color. To simplify the texturing process we choose $f$ as a combination of colors scaled by sinusoidal functions of the hyperspherical angles which go to black whenever $\theta_{i}$ is 0 or $\pi$. Examples of the resulting textures are visible in Figs. 1(b) and 1(c). The texture is then mapped onto individual particles given their current rotation and camera position. Further details are presented in Appendix B. The colormap used in four dimensions, as well as an additional explanation of hypersphere rotation and texturing, is available in a video in the Supplemental Material [22].

We have applied the previously described simulation and visualization method to three setups which are commonly simulated for 3D granular materials: the random packing of frictional hyperspheres, their uniaxial compression, and their flow on an inclined plane.

\section{RANDOM PACKING}

Particles are placed randomly in a simulation domain with a single wall whose normal vector points towards $e_{1}$, and periodic boundary conditions in all other spatial dimensions. The grains are left to relax under gravity acting along $-e_{1}$, until coming to rest forming a random packing on the wall [Figs. 2(a)-2(d)]. The thickness of this granular layer gives the solid volume fraction, and the number of contacts per grain yields an average coordination number, both of which are shown in Figs. 2(e) and 2(f). The interparticle friction coefficient $\mu_{g}$ is varied between 0 and 1 to explore the effect of friction on the packing fraction.

Both the random packing and the densest known volume packing fraction of balls decrease as the number of spatial dimensions increases [23]. In addition, higher friction coefficient between grains generally decreases the packing fraction in a given dimension, as the grains more easily interlock. Consequently, the measured volume fraction for frictional grains is significantly smaller than the one obtained for the random packing of hard spheres from [6].

Figure 3 explores the coordination number of the grains in different dimensions for the case $\mu_{g}=0.5$. The average coordination number shows an essentially linear increase with dimension in Fig. 3(a), which is markedly different from the much faster increase of the maximum number of hyperspheres that could potentially be in contact with a single particle (the kissing number). This suggests that the packing formation is mainly dictated by isostaticity and by the minimum number of grains necessary to maintain the stability of a single particle, which is itself given by $N$, and not by the boundary of

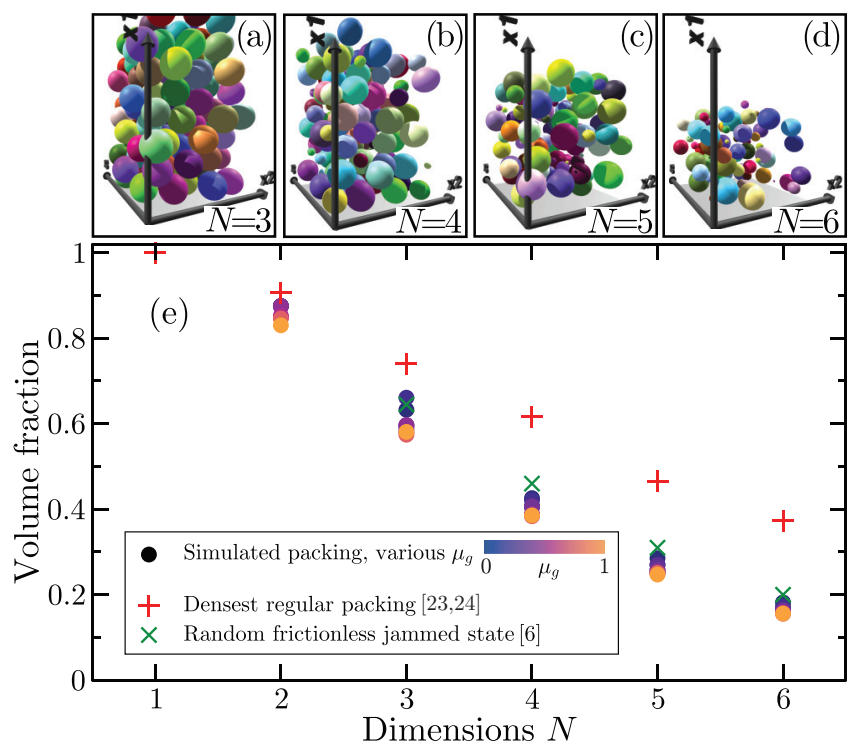

FIG. 2. Packing properties of dense granular media. [(a)-(d)] Particle visualizations of random packing in dimensions 3, 4, 5, and 6, respectively. The particles are colored randomly. (e) Volume fraction as a function of $N$ for the simulated random packing and for the densest known packing [23,24] and frictionless hypersphere packing [6].
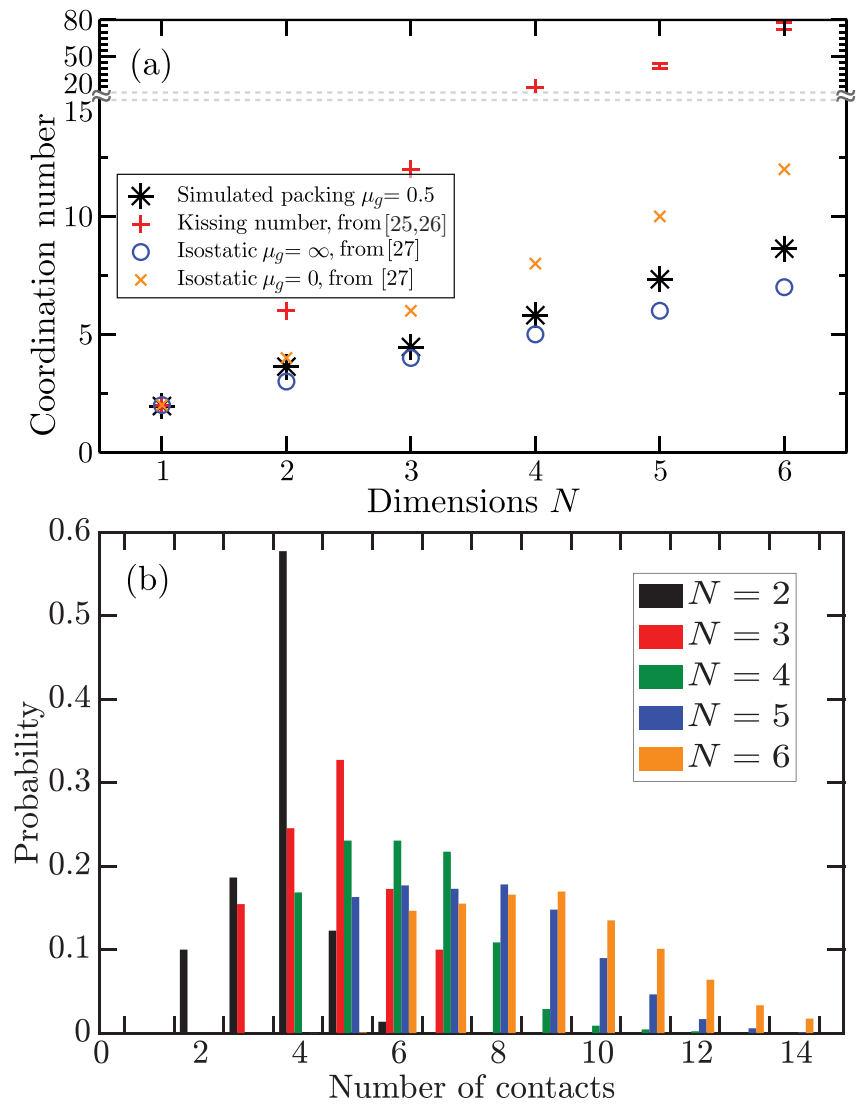

FIG. 3. (a) Coordination number as a function of $N$ for the simulated random packing with $\mu_{g}=0.5$ and maximum theoretical value of the coordination number $[25,26]$ (also known as the kissing number) and the isostatic packing bounds [27]. (b) Probability distribution of the number of contacts in different dimensions, $\mu_{g}=0.5$. 


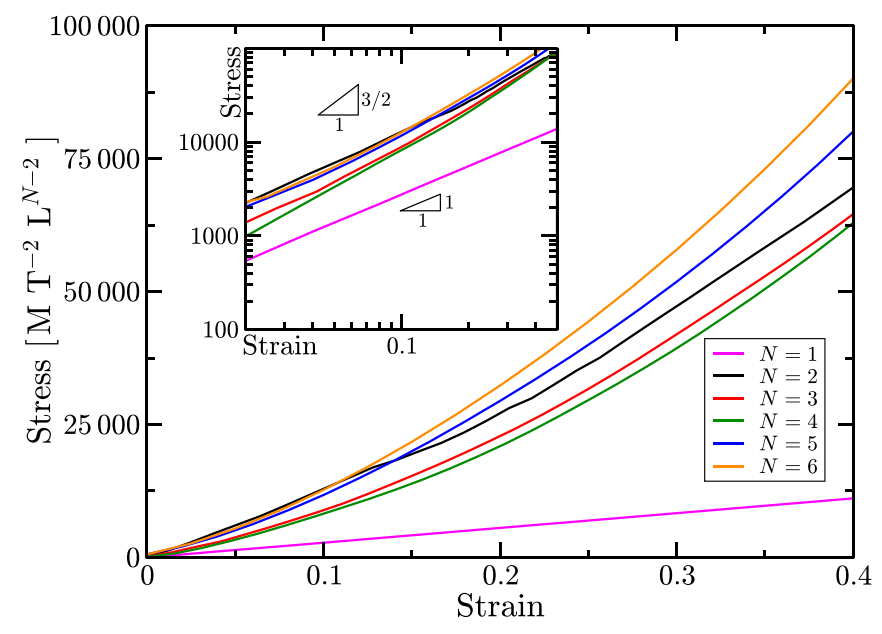

FIG. 4. Normal stress along the compression axis in uniaxial compression as a function of volumetric strain for different spatial dimensions. Inset: The same data on a logarithmic scale. A slope of 1 corresponds to the response of a linear elastic medium.

the $N$-ball. This is in agreement with the theoretical bounds of isostatic packing for spheres with no friction or infinite friction also indicated in Fig. 3(a). On an individual particle level, Fig. 3(b) shows that in all dimensions, a significant number of particles have more contacts than required from the isostaticity argument, which is expected to affect the overall mechanical properties of the packing. Note that since all the particles are in a stable static state, the minimum number of contacts for any grain in a given dimension is $N$.

\section{UNIAXIAL COMPRESSION}

The loading condition of uniaxial compression directly generalizes to higher dimensions. Starting from the packing condition described previously, a solid boundary is added above the grains and moved towards $-e_{1}$ at a constant velocity of $0.1\left[\mathrm{~L} \mathrm{~T}^{-1}\right]$. The friction coefficient between grain is kept constant at 0.5 during the uniaxial compression phase. The displacement when the moving boundary first touches the granular medium is taken to be a strain of 0 , and the normal stress $\sigma$ this applies to the boundary as a function of the volumetric strain $\varepsilon$ is shown in Fig. 4. These simulations do not show a clear trend with dimension number, suggesting similar mechanical properties of the bulk medium as a function of dimension. For $N=1$, the stress increases linearly with strain, indicating linear elasticity of the medium, which effectively corresponds to an array of linear springs. For $N>1$, however, the stress increases nonlinearly with strain, despite the contact law being an identical linear Hookean elastic law for all dimensions. The grain packing therefore significantly modifies the mechanical properties of the medium. It should be noted, however, that the use of linear spring interactions between grains may be physically inappropriate for higher dimensions, since it is well known that in dimension 3 a linear elastic material exhibits a nonlinear dependency for the normal contact of spheres (Hertzian model [28]).

The bulk stiffness of a 3D granular medium is known to significantly change with initial packing density $[29,30]$.

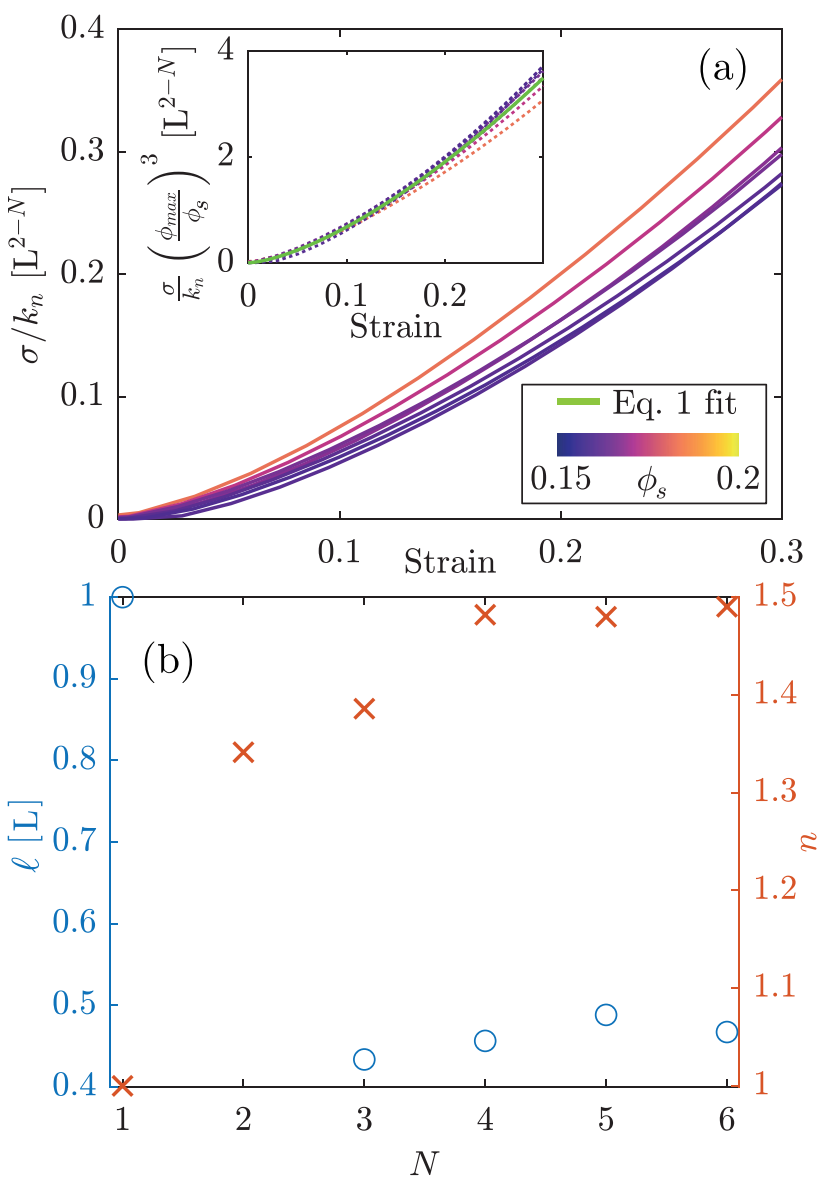

FIG. 5. (a) Example of scaled pressure versus strain in $N=6$ for uniaxial compression, with different initial packing volume fraction $\phi_{s}$. Inset: Pressure scaled by volume fraction dependency, and best fit with Eq. (1). (b) Coefficients $\ell$ and $n$ from Eq. (1) for various dimensions.

To study this effect more generally, we perform uniaxial compaction on packs prepared with different particle friction coefficient $\mu_{g}$, which create different initial packing densities as shown previously in Fig. 2(e). An example of this density dependence in dimension $N=6$ is shown in Fig. 5(a), where the stress $\sigma$ along $e_{1}$ scaled by the particle normal stiffness $k_{n}$ is shown for different initial volume fraction $\phi_{s}$. It is clear that the stress at a given strain is higher for samples starting at a higher volume fraction, which therefore appear stiffer. In dimension 3, this density dependency can be taken into account by including a $\phi_{s}^{3}$ dependency in the stiffness [31]. This scaling appears to fit these observations in dimensions higher than 1, as shown in the inset of Fig. 5(a) for $N=6$. Following this, we propose the following stress-strain dependency in our uniaxial compression of frictional hyperspheres:

$$
\frac{\sigma}{k_{n}}=\left(\frac{\phi_{s}}{\phi_{\max }}\right)^{3} \ell^{2-N} \varepsilon^{n},
$$

where $\phi_{\max }$ is the volume fraction of the densest regular packing in the considered dimension, and $\ell$ and $n$ are material parameters which depend on the dimension. Figure 5(b) shows the evolution of those parameters with dimension, indicating that $n$ tends to $3 / 2$ even with linear elastic contact 

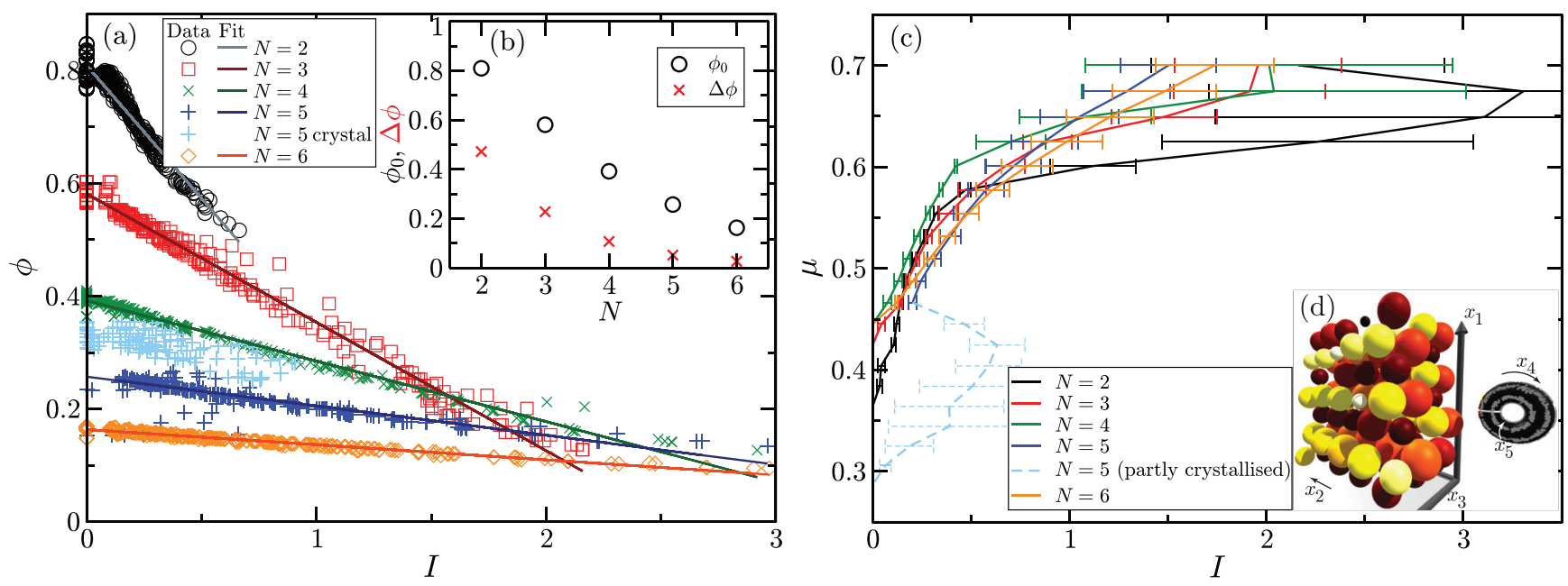

FIG. 6. Frictional behavior of hyperspheres during inclined plane flow. (a) Coarse-grained volume fraction $\phi$ as a function of inertial number $I$ for various dimensions $N$. (b) Values of best linear fit parameters to $\phi=\phi_{0}-\Delta \phi I$ as a function of $N$. (c) Friction coefficient $\mu$ as a function of inertial number $I$. The measured $I$ is averaged across the depth of the flow from the local coarse-grained values, and the error bars are the standard deviation around this mean estimation and do not take into account the uncertainty in the coarse-graining estimation (decreasing with increasing $N$ ). There are $\sim 15$ coarse-graining points in the flowing layer along $e_{1}$, using a window size $w=3 r$; see Appendix C for more details. (d) Example of a 5D crystalline state [shown in light blue in (a) and (c)]. Particle coloring indicates the location of the particle center in $x_{5}$. Particle centers in $x_{4}$ and $x_{5}$ are depicted on the torus in gray.

between the grains. The length $\ell$ is necessary to translate the stiffness at contact level, with units of $\left[\mathrm{M} \mathrm{T}^{-2}\right]$, to the stress, with units of $\left[\mathrm{M} \mathrm{T}^{-2} \mathrm{~L}^{2-N}\right]$. The value of $\ell$ is therefore 1 [L] for $N=1$, undetermined in $N=2$, and measured to be approximately 0.5 [L] for $N>2$.

\section{INCLINED PLANE FLOW}

Inclined plane flow also generalizes readily to higher dimensions for $N \geqslant 2$. In the first dimension, a solid boundary is located at $x_{1}=0$, and a gravity vector $\vec{g}$ is applied forming an angle $\alpha$ from $-\vec{e}_{1}$ towards $\vec{e}_{2}$, with $g=$ $10\left(-\cos \alpha \vec{e}_{1}+\sin \alpha \vec{e}_{2}\right)$. Again, every dimension other than the first has periodic boundaries. The initial domain size is $20 \times 5 \times 3.4 \times \cdots \times 3.4$ particle diameters in each spatial direction. The particles are initially positioned on an $N$ dimensional square lattice, with additional randomness added to their positions near the lattice points. The layer of particles nearest to the base at $x_{1}=0$ are kept fixed to act as a rough boundary. The grains are initially left to settle under gravity $g=-10 \vec{e}_{1}$. After consolidation, when the kinetic energy is close to zero, gravity is then tilted to $\alpha=35^{\circ}$, and the grains start to flow and eventually reach a steady state. Additional gravity angles are then investigated by decreasing $\alpha$ in $1^{\circ}$ steps and waiting for a steady state to be reached at each step, until eventually the grains come to rest. An example of such a flow can be seen in a Supplemental Material video [22].

The different regimes of flow are very similar for all dimensions, and consistent with previous observations in $N=2$ and 3, with a static pile at low angles and the development of a dense flow as $\alpha$ rises above a threshold value, which eventually leads to a supported flow [32] as $\alpha$ continues to increase where a layer of dense grains flows on top of grains in a gaseous state.
As has been done for 2D and 3D flows [33], it is convenient to summarize the flowing behavior of these materials by examining their frictional and volumetric behavior as a function of the inertial number, $I=2 r|\dot{\gamma}| \sqrt{\rho / P}$, which is well defined for all dimensions in this simple flow configuration. $\dot{\gamma}=\partial v_{2}^{\mathrm{cg}} / \partial x_{1}$ is the shear strain rate, which is obtained by discrete differentiation of the local coarse-grained velocity $v_{2}^{\text {cg }}$ along $e_{2} . P$ is the pressure in the material and is assumed to be the hydrostatic pressure, obtained by integrating the mass of grains from the surface of the flow given the volume fraction profile $\phi\left(x_{1}\right)$. The coarse-graining process used to obtain $v_{2}^{\mathrm{cg}}\left(x_{1}\right)$ and $\phi\left(x_{1}\right)$ is described in Appendix C.

By systematically varying the slope angle $\alpha$, it is possible to examine a range of $\mu=\tan (\alpha)$ and $I$ values, and a summary of the resulting information is shown in Fig. 6. The quasistatic volumetric concentrations measured from free-fall deposition above are recovered for low inertial numbers, and with increasing inertial number the flow expands. This expansion is characterized by the linear relation $\phi=\phi_{0}-\Delta \phi I$. Best-fit values of $\phi_{0}(N)$ and $\Delta \phi(N)$ are shown in Fig. 6(b), and both reduce with increasing dimension.

The $N=5$ dimension is clearly an outlier in this case, as at low inertial number we observe large values of $\phi$, significantly higher than the random packing observed in Fig. 2. This is attributed to the fact that the grains appear to crystallize as $\alpha$ is reduced, leading to a higher volume fraction in the crystallized phase. Figure $6(d)$ provides a visualization of this crystal. In addition to the 3D slice, particle locations in dimensions greater than three that are periodic can be mapped onto tori, two dimensions per torus. In the example shown in Fig. 6(d) of a 5D system, a single torus can represent the location of all additional dimensions. All particles are mapped onto the surface of the torus, with the current location of the observer marked by the two white intersecting circles. Both the 3D slice and the particles on the torus in Fig. 6(d) show clear alignment 
of the grains in preferential directions, quite different from the random packing in Fig. 2(c). The formation of this crystal is likely to depend strongly on the geometry of the simulation box, boundaries, and flow conditions, but a precise characterization of the dynamic emergence of such a crystal is left for further studies.

Kinematically, we find that all dimensions examined here behave in the same manner, with a similar $\mu(I)$ behavior, shown in Fig. 6(c). This suggests that the knowledge acquired from granular material behavior in two and three dimensions may be directly applicable in understanding frictional hypersphere packing and flows.

\section{CONCLUSION}

In conclusion, we have developed an extension to the discrete element method to simulate and visualize the behavior of frictional hyperspheres. This numerical technique has been used to simulate hyperspheres under free-fall consolidation, uniaxial compression, and inclined plane flow. This allowed the role of dimensionality in these setups to be uncovered and sheds new light on the use of $2 \mathrm{D}$ simulations to mimic $3 \mathrm{D}$ behavior. In particular, they validate the typical assumption that the stiffness and shear resistance of 2D granular media can be used to quantitatively predict 3D behavior.

Due to the inclusion of rotational degrees of freedom, it is now possible to simulate physically larger particles. This may be useful in describing various conformational problems, such as the entanglement and alignment of polymers, fibers, and sheets.

\section{ACKNOWLEDGMENT}

The authors thank I. Einav and P. Harrowell for fruitful discussions.

\section{APPENDIX A: SIMULATION METHOD}

\section{1. $\mathbb{N}$ dimensionality}

In this work we use the term hyperspace to refer to a Euclidean space $\mathbb{R}^{N}$ of $N \in \mathbb{N}$ dimensions, where $\mathbb{N}$ is the set of positive integers, with ortho-normal basis unit vectors $\vec{e}_{i}$ in each of the $N$ directions, such that a point in this space $\vec{x}$ can be defined as

$$
\vec{x}=x_{1} \vec{e}_{1}+x_{2} \vec{e}_{2}+\cdots+x_{i} \vec{e}_{i}+\cdots+x_{N} \vec{e}_{N},
$$

where $x_{i}$ are the components of this vector in each dimension $i$ from 1 to $N$.

A hypersphere is the direct generalization of a sphere in a hyperspace. The hypersphere is therefore the set of points located at a distance $r$ from the hypersphere center, such that for a hypersphere located at the origin, any point of the hypersphere satisfies

$$
x_{1}^{2}+x_{2}^{2}+\cdots+x_{N}^{2}=r^{2} .
$$

Similarly, an $N$-ball is the set of points inside a hypersphere, verifying $x_{1}^{2}+x_{2}^{2}+\cdots+x_{N}^{2} \leqslant r^{2}$. As it is common practice to use the term sphere for both a ball and its surface, in this work we use the term hypersphere to refer both to the surface of the $N$-ball and to the $N$-ball itself.
Now that we have the required building blocks to construct our simulation, we next need to define the equations of motion that are relevant to the discrete element method in order to compute the displacement of the hyperspheres.

\section{Linear momentum conservation}

The translational motion of a hypersphere can be directly solved by generalizing Newton's equations of motions. The velocity $\vec{v}$ of a particle in an $N$-dimensional space can be concisely written as

$$
\vec{v} \equiv \frac{d \vec{x}}{d t}=v_{1} \vec{e}_{1}+v_{2} \vec{e}_{2}+\cdots+v_{N} \vec{e}_{N} .
$$

Using this definition, we can directly generalize the conservation of linear momentum for a single particle to be

$$
m \frac{d \vec{v}}{d t}=\sum_{c} \vec{F}^{c}+m \vec{g}
$$

where $m$ is the mass of the particle, $\vec{v}$ its velocity, $\vec{F}^{c}$ the individual contact forces from neighboring particles, and $\vec{g}$ a body force acting on the particle, for example, a gravitational force. The sum is over all contacts $c$ of the particle.

The mass of a hypersphere in dimension $N$ of homogeneous density $\rho$ (expressed in $[\mathrm{M}][\mathrm{L}]^{-N}$ ) is known to be

$$
m=\rho V_{N}=\rho \frac{\pi^{N / 2}}{\Gamma\left(\frac{N}{2}+1\right)} r^{N}
$$

with $V_{N}=\frac{\pi^{N / 2}}{\Gamma\left(\frac{N}{2}+1\right)} r^{N}$ the volume of a hypersphere and $\Gamma$ the Gamma function.

Our discrete element simulation is dimensionless (unitless), therefore we will choose the diameter of our hypersphere as the length unit, $2 r=1[\mathrm{~L}]$, and the mass of such a hypersphere as the mass unit, $m=1[\mathrm{M}]$. The density $\rho$ of an individual hypersphere will therefore be different for different $N$ in order to maintain the same unit mass for the hyperspheres, as $V_{N}$ changes with $N$. The time unit [T] is set by the external force added to each of the particles, $\vec{g}=1\left[\mathrm{~L} \mathrm{~T}^{-2}\right]$. The equations of motion are solved using a Verlet algorithm with time step set to 0.0001 [T].

\section{Angular momentum conservation}

In three spatial dimensions, the angular momentum evolution law for a particle $p$ is typically written as

$$
I \frac{d \vec{\omega}}{d t}=\sum_{c} \overrightarrow{l^{c}} \times \overrightarrow{F^{c}}
$$

where $I$ is the moment of inertia of the particle, $\vec{\omega}$ the angular velocity vector, and $\overrightarrow{l^{c}} \times \overrightarrow{F^{c}}$ is the torque created by the contact force $\vec{F}^{c}$ applied on the particle at a contact point located at $\overrightarrow{l^{c}}$ relative to the center of mass of the particle.

This equation does not generalize directly to higher spatial dimensions, since the rotational velocity vector is only a pseudovector, rather than a true vector. As can be seen in Fig. 7, describing the rotational velocity of an object as a vector is applicable only in three spatial dimensions. In two dimensions, rotation can be described by a single scalar value, 

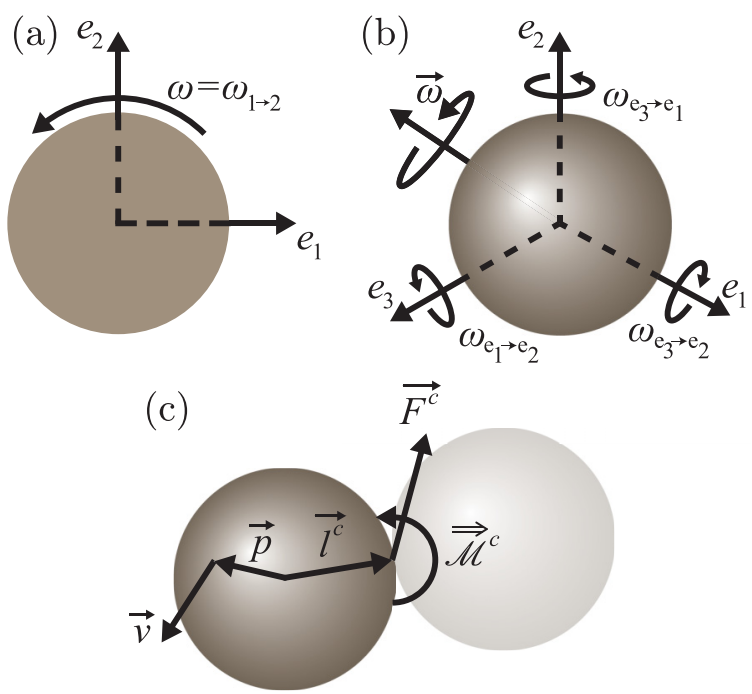

FIG. 7. Solid body rotation. (a) Definition of rotation rate $\omega$ in two dimensions. This is a rotation of the $2 \mathrm{D}$ object along a vector that is pointing out of the plane of rotation. (b) Definition of rotation rate $\vec{\omega}$ in three dimensions. This is a rotation of the $3 \mathrm{D}$ object about a 3D vector. (c) Definition of solid body rotation $\vec{\Omega}$ using Eq. (A7) and the contact between two hyperspheres.

which is therefore not a vector of the $2 \mathrm{D}$ space. A more general approach, general in arbitrary numbers of dimensions, is the concept of solid body rotation

$$
\vec{v}=-\vec{\Omega} \cdot \vec{p}
$$

where the velocity $\vec{v}$ of a point $\vec{p}$ pertaining to the solid body is given through the rotational velocity tensor $\vec{\Omega} . \vec{\Omega}$ is a skew-symmetric tensor whose components describe the motion of a point from one axis to another. For example, in three dimensions:

$$
\vec{\Omega}=\left(\begin{array}{ccc}
0 & \omega_{e_{1} \rightarrow e_{2}} & \omega_{e_{1} \rightarrow e_{3}} \\
\omega_{e_{2} \rightarrow e_{1}} & 0 & \omega_{e_{2} \rightarrow e_{3}} \\
\omega_{e_{3} \rightarrow e_{1}} & \omega_{e_{3} \rightarrow e_{2}} & 0
\end{array}\right)=\left(\begin{array}{ccc}
0 & \omega_{3} & -\omega_{2} \\
-\omega_{3} & 0 & \omega_{1} \\
\omega_{2} & -\omega_{1} & 0
\end{array}\right),
$$

and the associated rotation vector is $\vec{\omega}=\left(\omega_{1}, \omega_{2}, \omega_{3}\right)$. Since the rotational velocity tensor has $N(N-1) / 2$ degrees of freedom, it is clear that identification of the dual of $\vec{\Omega}$ with the rotational pseudovector $\vec{\omega}$ is possible only in three spatial dimensions.

From the solid body rotation Eq. (A7) it is straightforward to generalize the angular momentum conservation law by generalizing the vector product to the wedge product, leading to

$$
I \frac{d \vec{\Omega}}{d t}=\sum_{c} \overrightarrow{l^{c}} \wedge \vec{F}^{c}
$$

where the contact torque tensors $\overrightarrow{\mathcal{M}^{c}}=\overrightarrow{l^{c}} \wedge \vec{F}^{c}$ have components $\mathcal{M}_{i j}^{c}=l_{i}^{c} F_{j}^{c}-l_{j}^{c} F_{i}^{c}$.

These torques are skew-symmetric; therefore the skewsymmetric properties of $\vec{\Omega}$ are maintained upon time evolution through Eq. (A9). Finally, the generalization of the moment of inertia $I$ to any dimension is straightforward,

$$
I=\int \cdots \int_{\mathrm{N}-\text { ball }} \rho\|\vec{x}\| d x_{1} d x_{2} \cdots d x_{N}
$$

\section{Contact law}

In order to close the equations of motion, a contact law describing how particles interact with each other has to be chosen. For granular materials, the contact force resulting from the collision between two particles is usually modeled by an elastic normal and tangential contact, with a frictional cap on the tangential force, and viscous dissipation [16,28,34]. In this context, for two particles $i$ and $j$, the normal force $F_{n}$ in the direction normal to the contact, and the tangential force $F_{t}$ in the plane of contact, is given by

$$
\begin{gathered}
F_{n}=\left(k_{n} \delta_{n}^{a}-\gamma_{n} v_{n}\right), \\
F_{t}=\min \left(k_{t} \delta_{t}-\gamma_{t} v_{t}, \mu_{g} F_{n}\right),
\end{gathered}
$$

where $k_{n}, k_{t}, \gamma_{n}, \gamma_{t}, \mu_{g}$ are the normal and tangential elastic constants, normal and tangential viscosity, and friction coefficient, respectively; $v_{n}$ and $v_{t}$ are the relative normal and tangential velocity between the grains; $\delta_{n}$ and $\delta_{t}$ are the normal and tangential interpenetration, the latter taking into account the history of the contact. The exponent $a$ is 1 for a Hookean contact [34] (known to be physically realistic in two dimensions) and 3/2 for a Hertzian contact [28] (equivalently in three dimensions). The derivation of the contact force resulting from the contact of two elastic hyperspheres being beyond the scope of this paper, and in order to limit the demand on computational power, we use $a=1$ independent of the number of spatial dimensions in our simulations. Additionally, the material contact parameters are kept constant across all our simulations with $k_{n}=2 \times 10^{5}\left[\mathrm{MT}^{-2}\right]$, $k_{t}=8 \times 10^{4}\left[\mathrm{MT}^{-2}\right], \gamma_{n}=\gamma_{t}=75\left[\mathrm{MT}^{-1}\right]$ corresponding to a restitution coefficient of approximately 0.8 , and $\mu_{g}=0.5$.

The numerical computation of the contact forces follows [35], with the only difference being that the velocities on both sides of the contact point must be computed using Eq. (A7).

\section{Discrete element solver}

The higher dimensional discrete element method algorithm described above is implemented in the NDDEM Solver. This parallelized code was used to produce the results contained in this work. It can simulate particles in any number of dimension $N>0$, limited only by computing capabilities. Due to the nature of how hyperspheres fill hyperspace, in practical applications of dense granular flows such as those examined here, we are limited to $N \lesssim 10$ for computational reasons. In the NDDEM Particle Visualisation Tool, users can explore this space with either a keyboard or mouse combination, or in virtual reality, where hand controls can be used to navigate in up to seven dimensions. This is implemented using the Javascript library three.js [36], and live versions of precalculated DEM data can be accessed at Ref. [21]. 


\section{APPENDIX B: ORIENTATION TRACKING}

Although it is not strictly necessary to track the hypersphere orientation over time, as the laws of motion depend only on the rotational velocity and not the orientation itself, it is often useful to do so, in particular for visualization purposes. An orthonormal tensor $\vec{A}$ representing an orientation reference frame is therefore attached to each hypersphere, and is evolved over time according to Eq. (A7), leading to

$$
\frac{d \vec{A}}{d t}=-\vec{\Omega} \vec{A}, \quad \vec{A}(t=0)=\overrightarrow{1}_{N}
$$

with $\overrightarrow{1}_{N}$ the identity matrix in dimension $N$. Although Eq. (B1) has an analytical solution for constant $\vec{\Omega}$ through matrix exponentiation, it needs to be solved numerically for varying angular velocities. Time integration schemes do not necessarily maintain the orthonormality of $\vec{A}$, which therefore would lose this property over time due to numerical error accumulation. As $\vec{A}$ is not involved in solving the dynamics, we decided against using an advanced numerical integration that would have helped to preserve orthonormality [37], and instead simply use a Gram-Schmidt process to orthonormalize $\vec{A}$ at each time step.

\section{Particle texturing}

To visualize the orientation of a sphere, it is convenient to attach a texture to the surface of the sphere which rotates with the sphere. A similar approach is followed to visualize the orientation of a hypersphere on its 3D intersection in our visualization software. In contrast to conventional UV mapping for $3 \mathrm{D}$ objects, it is not possible to generate fixed textures which rotate with the object, as the boundary of a hypersphere is in general not a 2D texture.

We define the boundary of the hyperspheres using hyperspherical coordinates comprising $N-1$ angles $\varphi, \theta_{1}, \theta_{2}, \ldots, \theta_{N-2}$, with the angles $\varphi \in[0,2 \pi]$ and $\theta_{i} \in$ $[0, \pi]$. A "texturing" function maps this coordinate space to the color space.

The process to texture a hypersphere for visualization in the $3 \mathrm{D}$ space described above is therefore the following. First, we define a coordinate system $u, v$ on the 3-ball surface which we want to color. By discretizing these coordinates into $n$ values each, we recover a set of $3 \mathrm{D}$ points in $x_{1}, x_{2}$, and $x_{3}$. The locations of the points in the remaining $N-3$ dimensions are set by our viewpoint. As these points are on the boundary of the rotated hypersphere, we must bring them back to their nonrotated frame to be able to calculate what color they each correspond to, which can be achieved by multiplying them by $A^{-1}=\overrightarrow{A^{T}}$. Finally we can color them using an appropriate color mapping.

Since the space of colors has effectively only three dimensions [for example, (red, green, blue), or (hue, saturation, value)], there is no bijection from the $N-1$-dimensional hypersphere boundary to the color space for $N>4$, therefore some points of the hypersphere boundary must have the same color for $N>4$.

\section{APPENDIX C: COARSE GRAINING}

In order to obtain continuum fields from particle information, it is often necessary in discrete element simulations to coarse grain the numerical data, i.e., to perform spatial averaging. We follow the established state-of-the-art 3D coarse-graining method [38-40] using a generalized $N$-dimensional Lucy windowing function $\mathcal{W}$ of width $w$, satisfying $\int_{\mathbb{R}^{N}} \mathcal{W} d \vec{x}=1$, and with value at a distance $d$ from the coarse-graining point of

$$
\mathcal{W}\left(\frac{d}{w}\right)= \begin{cases}\frac{1}{C_{N}} \times\left(-3\left(\frac{d}{w}\right)^{4}+8\left(\frac{d}{w}\right)^{3}-6\left(\frac{d}{w}\right)^{2}+1\right), & \text { if } d<w \\ 0, & \text { otherwise }\end{cases}
$$

with

$$
C_{N}=V_{N} N\left(\frac{-3}{N+4}+\frac{8}{N+3}-\frac{6}{N+2}+\frac{1}{N}\right) .
$$

However, since this paper focuses on flow with periodic boundaries in all directions but one, we here use the 1D Lucy function in the nonperiodic direction and all the particles in the periodic directions. $\overrightarrow{x^{\mathrm{cg}}}$

This leads to the usual expression for the volume fraction $\phi$, and the coarse-grained velocity $\overrightarrow{v^{\mathrm{cg}}}$ at the coarse-graining point

$$
\begin{gathered}
\phi\left(\overrightarrow{x^{\mathrm{cg}}}\right)=\frac{1}{\rho} \sum_{p} \mathcal{W}\left(\left|\overrightarrow{x^{p}}-\overrightarrow{x^{\mathrm{cg}}}\right|\right) m^{p}, \\
\rho \phi\left(\overrightarrow{x^{\mathrm{cg}}}\right) \overrightarrow{v^{\mathrm{cg}}}\left(\overrightarrow{x^{\mathrm{cg}}}\right)=\sum_{p} \mathcal{W}\left(\left|\overrightarrow{x^{p}}-\overrightarrow{x^{\mathrm{cg}}}\right|\right) m^{p} \overrightarrow{v^{p}}
\end{gathered}
$$

where $\rho$ is the particle density as defined above, and the sum is over all the particles $p$ at location $\overrightarrow{x^{p}}$ with mass $m^{p}$ and velocity $\overrightarrow{v^{p}}$. In a similar manner it is possible to extract additional fields such as the stresses, angular velocities, 
moments, kinetic energies, etc., with any number of dimensions. These higher dimensional coarse-grained fields can be visualized in the NDDEM Coarse Graining Visualisation Tool.
[1] A. Einstein, Die Grundlage der allgemeinen Relativitätstheorie, Ann. Phys. 354, 769 (1916).

[2] J. M. Maldacena, The large-N limit of superconformal field theories and supergravity, Int. J. Theor. Phys. 38, 1113 (1999).

[3] P. G. Freund, Superstrings from 26 dimensions?, Phys. Lett. B 151, 387 (1985).

[4] P. De Wolff, The pseudo-symmetry of modulated crystal structures, Acta Crystallogr. A 30, 777 (1974).

[5] N. J. Sloane, The packing of spheres, Sci. Am. 250, 116 (1984).

[6] M. Skoge, A. Donev, F. H. Stillinger, and S. Torquato, Packing hyperspheres in high-dimensional Euclidean spaces, Phys. Rev. E 74, 041127 (2006).

[7] P. Charbonneau, A. Ikeda, G. Parisi, and F. Zamponi, Glass Transition and Random Close Packing Above Three Dimensions, Phys. Rev. Lett. 107, 185702 (2011).

[8] C. E. Shannon, A mathematical theory of communication, Bell Syst. Tech. J. 27, 379 (1948).

[9] P. Delsarte, Bounds for unrestricted codes, by linear programming, Philips Res. Rep. 27, 272 (1972).

[10] G. M. Crippen, Conformational analysis by energy embedding, J. Comput. Chem. 3, 471 (1982).

[11] R. C. van Schaik, H. J. Berendsen, A. E. Torda, and W. F. van Gunsteren, A structure refinement method based on molecular dynamics in four spatial dimensions, J. Mol. Biol. 234, 751 (1993).

[12] G. Bottegoni, I. Kufareva, M. Totrov, and R. Abagyan, Fourdimensional docking: A fast and accurate account of discrete receptor flexibility in ligand docking, J. Med. Chem. 52, 397 (2009).

[13] J. Michels and N. Trappeniers, Dynamical computer simulations on hard hyperspheres in four-and five-dimensional space, Phys. Lett. A 104, 425 (1984).

[14] L. Lue, Collision statistics, thermodynamics, and transport coefficients of hard hyperspheres in three, four, and five dimensions, J. Chem. Phys. 122, 044513 (2005).

[15] L. Lue, M. Bishop, and P. A. Whitlock, The fluid to solid phase transition of hard hyperspheres in four and five dimensions, J. Chem. Phys. 132, 104509 (2010).

[16] P. A. Cundall and O. D. L. Strack, A discrete numerical model for granular assemblies, Géotechnique 29, 47 (1979).

[17] T. Weinhart, L. Orefice, M. Post, M. P. van Schrojenstein Lantman, I. F. Denissen, D. R. Tunuguntla, J. Tsang, H. Cheng, M. Y. Shaheen, H. Shi et al., Fast, flexible particle simulationsAn introduction to MercuryDPM, Comput. Phys. Commun. 249, 107129 (2020).

[18] U. Ayachit, The ParaView Guide: A Parallel Visualization Application (Kitware, Inc., Clifton Park, NY, 2015).

[19] W. Humphrey, A. Dalke, and K. Schulten, VMD-visual molecular dynamics, J. Mol. Graphics 14, 33 (1996).

[20] Blender Online Community, Blender-A $3 D$ modelling and rendering package, Blender Foundation, Blender Institute, Amsterdam (2020).

[21] https://github.com/Franzzzzzzzz/NDDEM.

[22] See Supplemental Material at http://link.aps.org/supplemental/ 10.1103/PhysRevE.103.052901 for a video describing hypersphere rendering from hyperspaces to 3D space, one of particle rotation in higher dimensional spaces, and orientation mapping, rendering, and visualization, and one with examples of inclined place flows in $N$ from 3 to 6 .

[23] T. C. Hales and S. P. Ferguson, A formulation of the Kepler conjecture, Discrete Comput. Geom. 36, 21 (2006).

[24] J. H. Conway and N. J. A. Sloane, Sphere Packings, Lattices and Groups, 3rd edition, Volume 290 in Grundlehren der mathematischen Wissenschaften (Springer ed. New York, 1999).

[25] A. M. Odlyzko and N. J. Sloane, New bounds on the number of unit spheres that can touch a unit sphere in $n$ dimensions, J. Comb. Theory A 26, 210 (1979).

[26] O. R. Musin, The kissing number in four dimensions, Ann. Math. 168, 1 (2008).

[27] C. Song, P. Wang, and H. A. Makse, A phase diagram for jammed matter, Nature (London) 453, 629 (2008).

[28] H. Hertz, Ueber die Berührung fester elastischer Körper, Journal für die reine und angewandte Mathematik 92, 156 (1882).

[29] G. Viggiani and J. H. Atkinson, Stiffness of fine-grained soil at very small strains, Géotechnique 45, 249 (1995).

[30] B. O. Hardin and W. L. Black, Sand stiffness under various triaxial stresses, J. Soil Mech. Found. Div. 92, 27 (1966).

[31] M. B. Rubin and I. Einav, A large deformation breakage model of granular materials including porosity and inelastic distortional deformation rate, Int. J. Eng. Sci. 49, 1151 (2011).

[32] N. Brodu, R. Delannay, A. Valance, and P. Richard, New patterns in high-speed granular flows, J. Fluid Mech. 769, 218 (2015).

[33] GDR MiDi, On dense granular flows, Eur. Phys. J. E 14, 341 (2004).

[34] R. Hooke, Lectures De Potentia Restitutiva, or of Spring Explaining the Power of Springing Bodies (John Martyn, 1678).

[35] S. Luding, Cohesive, frictional powders: Contact models for tension, Granular Matter 10, 235 (2008).

[36] https://threejs.org/.

[37] D. J. Higham, Runge-Kutta type methods for orthogonal integration, Appl. Numer. Math. 22, 217 (1996).

[38] M. Babic, Average balance equations for granular materials, Int. J. Eng. Sci. 35, 523 (1997).

[39] I. Goldhirsch, Stress, stress asymmetry and couple stress: From discrete particles to continuous fields, Granular Matter 12, 239 (2010).

[40] T. Weinhart, A. R. Thornton, S. Luding, and O. Bokhove, From discrete particles to continuum fields near a boundary, Granular Matter 14, 289 (2012). 